\title{
Methods for parallel-detection-based ultrasound-modulated optical tomography
}

\author{
Jun Li and Lihong V. Wang
}

\begin{abstract}
The research reported here focuses on ultrasound-modulated optical tomography based on parallel speckle detection. Four methods were investigated for signal acquisition and analysis, in which laser speckle statistics was applied. The methods were compared with the previously used four-phase method in the imaging of all-biological-tissue samples, in which the buried objects were also biological tissues. The image quality obtained with these methods was comparable with that obtained with the four-phase method; in addition, these methods have advantages in reducing acquisition time and improving the signal-to-noise ratio. (C) 2002 Optical Society of America
\end{abstract}

OCIS codes: $170.3880,120.6150,110.7050,110.7170$

\section{Introduction}

Because of the obvious desirability of its noninvasive and non-ionizing characteristics for detecting cancers at an early stage, the optical imaging of biological tissues has been the focus of much research in recent years. The contrast mechanism of optical imaging is based on the difference in optical properties (such as absorption or scattering) between abnormal tissues and the surrounding normal tissues. Several optical imaging techniques, such as time-resolved optical imaging, frequency-domain optical imaging, and optical coherence tomography, have been studied previously. However, because biological tissues are optically turbid media, light is strongly scattered inside tissues and the scattered light provides poor localization information. To overcome this problem, hybrid methods that combine optical and ultrasonic techniques have been explored. These methods include photoacoustic imaging, ${ }^{1-3}$ sonoluminescent tomography, ${ }^{4}$ and ultrasound-modulated optical tomography. ${ }^{5-8}$ Because ultrasonic waves are scattered less than light waves in biological tissues, they can be used to provide spatial information directly for imaging. In ultrasound-modulated optical tomography, an ultra-

The authors are with the Optical Imaging Laboratory, Biomedical Engineering Program, Texas A\&M University, 3120 TAMU, College Station, Texas 77843-3120. L. V. Wang's e-mail address is LWang@tamu.edu.

Received 13 September 2001; revised manuscript received 12 December 2001.

0003-6935/02/102079-06\$15.00/0

(C) 2002 Optical Society of America sonic wave is focused into tissue to modulate the light passing through the ultrasonic zone. The modulated or tagged light carries the ultrasonic frequency and, therefore, can be discriminated from the background unmodulated light, and its origins can be derived from the position of the ultrasonic column inside the tissue.

Ultrasound-modulated optical tomography has been studied by several research groups. Marks et al. ${ }^{5}$ investigated the combination of pulsed ultrasound and laser light and detected an ultrasound-modulated optical signal in a homogeneous turbid medium. Wang et al.6,7 developed ultrasound-modulated optical tomography with continuous-wave ultrasound and obtained images in tissue-simulating turbid media. Wang and $\mathrm{Ku}^{9}$ used a frequency-swept technique to achieve controllable spatial resolution along the ultrasonic axis. Leveque et al. ${ }^{10}$ developed a parallel speckle detection, in which a CCD camera worked as a detector array and the signal-to-noise ratio (SNR) was improved by averaging the signals from all of the CCD pixels. They obtained one-dimensional (1D) images of biological tissue. Yao and Wang ${ }^{11}$ subsequently obtained two-dimensional (2D) images of multiple objects buried in biological tissue. Yao et al. ${ }^{12}$ further developed the technique by combining the parallel-detection and the frequency-swept techniques and obtained 2D images of biological tissues in which one dimension was along the ultrasonic axis. By use of parallel detection, Leveque-Fort ${ }^{13}$ obtained three-dimensional images in biological tissue. Besides the imaging in transmission configurations, imaging in reflection configurations has also been studied by Lev et al., ${ }^{14}$ Granot et al., ${ }^{15}$ and Leveque-Fort et al. ${ }^{16}$ In addition, 
Leutz and Maret ${ }^{17}$ and Mahan et al. ${ }^{18}$ investigated selected mechanisms of ultrasonic modulation of light in scattering media. More recently, Wang ${ }^{19,20}$ developed comprehensive analytic and Monte Carlo models that accounted for all of the contributing mechanisms, and they were able to evaluate their respective contributions.

The parallel speckle detection ${ }^{10-13,16}$ is, so far, the most efficient technique for ultrasound-modulated optical tomography. Because four acquisitions are needed to recover the modulated signals and each acquisition has to collect sufficient photons to maintain enough SNR, the long acquisition time may lead to speckle decorrelation. In this paper, we investigate four methods for data acquisition and analysis: a three-phase method, two two-phase methods, and a cross-correlation method. The two-phase methods are similar to but different from the approach by Selb et al. ${ }^{21}$ Both Selb et al. and our group used twoacquisition detection schemes independently with different treatments. We proposed two methods for the two-acquisition detection, and more significantly, we compared the images with the commonly used four-phase method. The purpose of the study is, by a careful comparison of the methods, to find efficient ways for parallel speckle detection. In our experiments, we obtained 2D images of all-biological-tissue samples in which the objects of interest were also biological tissues. The four methods produced image qualities comparable with that of the fouracquisition method but had advantages in shortening the acquisition time and improving the SNR.

\section{Methods of Signal Acquisition and Analysis}

\section{A. Four-Phase Method}

In the parallel-detection method, a source-synchronized lock-in technique is applied and a CCD camera is used as a detector array with each pixel acting as a single detector. The speckle size on the CCD surface is adjusted to match the pixel size. The signal in a single pixel can be expressed as

$$
I=I_{\mathrm{dc}}+I_{\mathrm{ac}} \cos \left(\phi_{\mathrm{s}}+\phi\right),
$$

where $I_{\mathrm{dc}}$ is the background intensity; $I_{\mathrm{ac}}$ is the signal intensity related to the modulated ac signal; $\phi_{s}$ is the initial phase of the speckle; and $\phi$ is the phase delay of the signal applied to the ultrasonic transducer relative to the signal applied to the laser.

Traditionally in parallel detection, four acquisitions were made to recover the modulation signals, ${ }^{10-13}$ where the four sequential acquisitions have phase delays of $0^{\circ}, 90^{\circ}, 180^{\circ}$, and $270^{\circ}$, respectively. The signals from the four acquisitions are as follows:

$$
\begin{aligned}
& I_{1}=I_{\mathrm{dc}}+I_{\mathrm{ac}} \cos \phi_{s}, \\
& I_{2}=I_{\mathrm{dc}}+I_{\mathrm{ac}} \sin \phi_{s}, \\
& I_{3}=I_{\mathrm{dc}}-I_{\mathrm{ac}} \cos \phi_{s}, \\
& I_{4}=I_{\mathrm{dc}}-I_{\mathrm{ac}} \sin \phi_{s} .
\end{aligned}
$$

From Eqs. (2)-(5), we obtain

$$
\begin{aligned}
& I_{\mathrm{dc}}=\left(I_{1}+I_{2}+I_{3}+I_{4}\right) / 4, \\
& I_{\mathrm{ac}}=\left[\left(I_{1}-I_{3}\right)^{2}+\left(I_{2}-I_{4}\right)^{2}\right]^{1 / 2} .
\end{aligned}
$$

We define quantity $M=I_{\mathrm{ac}} / I_{\mathrm{dc}}$ as the modulation depth, which reflects the local optical and ultrasonic properties. After averaging the modulation depths obtained from all of the CCD pixels, we take the average $\langle M\rangle$ as the imaging signal corresponding to the current location of the ultrasonic column. By comparing experimental data of $\left\langle I_{\mathrm{ac}} / I_{\mathrm{dc}}\right\rangle$ and $\left\langle I_{\mathrm{ac}}\right\rangle /$ $\left\langle I_{\mathrm{dc}}\right\rangle$, we found there was little difference, less than $0.1 \%$, between the values. In practice, we take $\langle M\rangle$ $\approx\left\langle I_{\mathrm{ac}}\right\rangle /\left\langle I_{\mathrm{dc}}\right\rangle$.

\section{B. Three-Phase Method}

In this method, we simply reduce the number of acquisitions from four to three by making acquisitions at phase delays of $0^{\circ}, 90^{\circ}, 180^{\circ}$ or other combinations of three phases. From Eqs. (2)-(5), we get

$$
\begin{aligned}
& I_{\mathrm{dc}}=\left(I_{1}+I_{3}\right) / 2, \\
& I_{\mathrm{ac}}=\left[\left(I_{1}-I_{3}\right)^{2}+\left(2 I_{2}-I_{1}-I_{3}\right)^{2}\right]^{1 / 2}
\end{aligned}
$$

for the combination of $0^{\circ}, 90^{\circ}$, and $180^{\circ}$, or

$$
\begin{aligned}
& I_{\mathrm{dc}}=\left(I_{2}+I_{4}\right) / 2, \\
& I_{\mathrm{ac}}=\left[\left(I_{2}-I_{4}\right)^{2}+\left(2 I_{3}-I_{2}-I_{4}\right)^{2}\right]^{1 / 2}
\end{aligned}
$$

for the combination of $90^{\circ}, 180^{\circ}$, and $270^{\circ}$.

In this case, the CCD images can be obtained as in the four-phase method. This method requires three acquisitions and consumes, therefore, three fourths of the acquisition time required by the conventional four-phase method.

\section{Two-Phase Method I}

In this method, we further reduce the acquisitions from three to two, which can be done in either of two ways. In the first method, two acquisitions with a phase difference of $180^{\circ}$ are selected, such as $0^{\circ}$ and $180^{\circ}$. From Eqs. (2) and (4), one can obtain

$$
I_{\mathrm{dc}}=\left(I_{1}+I_{3}\right) / 2 \text {. }
$$

Subtracting Eq. (4) from Eq. (2) and squaring the result generates

$$
4 I_{\mathrm{ac}}{ }^{2} \cos ^{2} \phi_{s}=\left(I_{1}-I_{3}\right)^{2} .
$$

Based on the speckle statistics that speckles have random phases, the average of $\cos 2 \phi_{s}$ over the speckle field should be zero, and the average of $\cos ^{2} \phi_{s}$ over the speckle field should be $1 / 2^{22}$ :

$$
\left\langle\cos ^{2} \phi_{s}\right\rangle=1 / 2 \text {. }
$$

Averaging the variables in Eq. (13) for all CCD pixels and applying Eq. (14), we have

$$
\left\langle I_{\mathrm{ac}}{ }^{2}\right\rangle=\left\langle\left(I_{1}-I_{3}\right)^{2}\right\rangle / 2 .
$$


In the paper by Selb et al. ${ }^{21}$ the authors extracted the item involving $I_{\mathrm{dc}}$ by subtracting two equations that were similar to Eq. (2) and Eq. (4), taking the absolute value of the difference, and using the relation$\operatorname{ship}\left\langle\left|\sin \phi_{s}\right|\right\rangle=2 / \pi$. Our treatment is similar to but different from theirs.

To obtain the imaging signal $\langle M\rangle$, approximation $\left\langle I_{\mathrm{ac}}\right\rangle^{2} \approx\left\langle I_{\mathrm{ac}}{ }^{2}\right\rangle$ is applied, which is valid when variance $\sigma_{\mathrm{ac}}{ }^{2}$ (i.e., $\left\langle I_{\mathrm{ac}}{ }^{2}\right\rangle-\left\langle I_{\mathrm{ac}}{ }^{2}\right)$ is small compared with $\left\langle I_{\mathrm{ac}}{ }^{2}\right\rangle$ and $\left\langle I_{\mathrm{ac}}\right\rangle^{2}$.

$$
\langle M\rangle \approx\left\langle I_{\mathrm{ac}}{ }^{2}\right\rangle^{1 / 2} /\left\langle I_{\mathrm{dc}}\right\rangle=\left[\left\langle\left(I_{1}-I_{3}\right)^{2}\right\rangle / 2\right]^{1 / 2} /\left\langle\left(I_{1}+I_{3}\right) / 2\right\rangle .
$$

Similarly, for the acquisitions with phase delays of $90^{\circ}$ and $270^{\circ}$, the imaging signal is

$$
\langle M\rangle \approx\left\langle I_{\mathrm{ac}}{ }^{2}\right\rangle^{1 / 2} /\left\langle I_{\mathrm{dc}}\right\rangle=\left[\left\langle\left(I_{2}-I_{4}\right)^{2}\right\rangle / 2\right]^{1 / 2} /\left\langle\left(I_{2}+I_{4}\right) / 2\right\rangle .
$$

\section{Two-Phase Method II}

Alternatively, we can process the signals from the two-phase acquisitions by squaring Eq. (2) and averaging each variable over all the CCD pixels. Applying the statistical properties, that is, Eq. (14), we obtain

$$
\begin{aligned}
& \left\langle I_{1}{ }^{2}\right\rangle=\left\langle I_{\mathrm{dc}}{ }^{2}\right\rangle+\left\langle I_{\mathrm{ac}}{ }^{2}\right\rangle / 2, \\
& \langle M\rangle \approx\left(\left\langle I_{\mathrm{ac}}{ }^{2}\right\rangle /\left\langle I_{\mathrm{dc}}{ }^{2}\right\rangle\right)^{1 / 2}=\left[2\left(\left\langle I_{1}{ }^{2}\right\rangle /\left\langle I_{\mathrm{dc}}{ }^{2}\right\rangle-1\right)\right]^{1 / 2},
\end{aligned}
$$

or

$$
\langle M\rangle \approx\left(\left\langle I_{\mathrm{ac}}{ }^{2}\right\rangle /\left\langle I_{\mathrm{dc}}{ }^{2}\right\rangle\right)^{1 / 2}=\left[2\left(\left\langle I_{3}{ }^{2}\right\rangle /\left\langle I_{\mathrm{dc}}{ }^{2}\right\rangle-1\right)\right]^{1 / 2},
$$

where $I_{\mathrm{dc}}=\left(I_{1}+I_{3}\right) / 2$ and approximation $\left\langle I_{\mathrm{ac}} / I_{\mathrm{dc}}\right\rangle$ $\approx\left(\left\langle I_{\mathrm{ac}}{ }^{2}\right\rangle /\left\langle I_{\mathrm{dc}}{ }^{2}\right\rangle\right)^{1 / 2}$ are applied. Modulation depth measured in a CCD pixel can be expressed as $I_{\mathrm{ac}} / I_{\mathrm{dc}}$ $=\left\langle I_{\mathrm{ac}} / I_{\mathrm{dc}}\right\rangle+\delta$, where $\delta(<0$, or, $>0)$ is the deviation of modulation depth $I_{\mathrm{ac}} / I_{\mathrm{dc}}$ to the mean $\left\langle I_{\mathrm{ac}} / I_{\mathrm{dc}}\right\rangle$. By squaring the equation, averaging over all CCD pixels, and ignoring the item involving $\langle\delta\rangle$ and the item involving $\left\langle\delta^{2}\right\rangle$, we obtain the above approximation.

Similarly, for the combination of $90^{\circ}$ and $270^{\circ}$ phase delays, we have

$$
\langle M\rangle \approx\left(\left\langle I_{\mathrm{ac}}{ }^{2}\right\rangle /\left\langle I_{\mathrm{dc}}{ }^{2}\right\rangle\right)^{1 / 2}=\left[2\left(\left\langle I_{2}{ }^{2}\right\rangle /\left\langle I_{\mathrm{dc}}{ }^{2}\right\rangle-1\right)\right]^{1 / 2},
$$

or

$$
\langle M\rangle \approx\left(\left\langle I_{\mathrm{ac}}{ }^{2}\right\rangle /\left\langle I_{\mathrm{dc}}{ }^{2}\right\rangle\right)^{1 / 2}=\left[2\left(\left\langle I_{4}{ }^{2}\right\rangle /\left\langle I_{\mathrm{dc}}{ }^{2}\right\rangle-1\right)\right]^{1 / 2},
$$

where $I_{\mathrm{dc}}=\left(I_{2}+I_{4}\right) / 2$. The two-phase methods would reduce by half the acquisition time compared with that of the four-phase method.

\section{E. Cross-Correlation Method}

In addition, we applied the correlation relationship for signal processing. With the use of the aforementioned phase-delay parallel detection, we calculate the cross-correlation coefficients between the speckle patterns acquired with different phase delays. Because of the different phase delays, the speckle patterns will differ. The decorrelation of the speckle

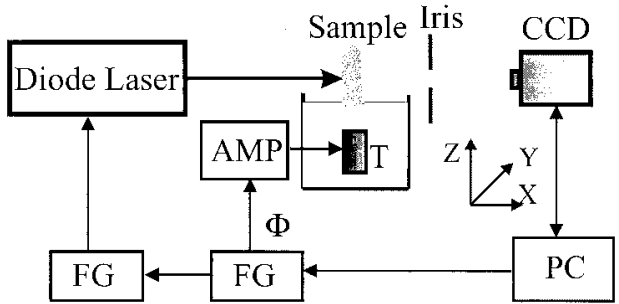

Fig. 1. Experimental setup: T, transducer; AMP, power amplifier; FG, function generator; PC, personal computer.

pattern between the two acquisitions reflects the effect of ultrasonic modulation if the decorrelation caused by Brownian motion is negligible within the acquisition time period. The cross-correlation coefficient between the $m$ th and $n$th speckle patterns is calculated as

$$
\begin{aligned}
R_{m, n}= & \sum_{i=1}^{N}\left(I_{m, i}-\left\langle I_{m}\right\rangle\right)\left(I_{n, i}-\left\langle I_{n}\right\rangle\right) \mid \\
& {\left[\sum_{i=1}^{N}\left(I_{m, i}-\left\langle I_{m}\right\rangle\right)^{2} \sum_{i=1}^{N}\left(I_{n, i}-\left\langle I_{n}\right\rangle\right)^{2}\right]^{1 / 2}, }
\end{aligned}
$$

where $m, n=1,2,3,4 ; N$ is the number of pixels of the CCD camera; $I_{m, i}$ and $I_{n, i}$ are the signal intensities in the $i$ th pixel in the $m$ th and $n$th speckle patterns, respectively; and $\left\langle I_{m}\right\rangle$ and $\left\langle I_{n}\right\rangle$ are the average signal intensities of the $m$ th and $n$th speckle patterns, respectively. The quantity $1-R_{m, n}$ is taken as the imaging signal. Because only two acquisitions are required for correlation processing, the acquisition time is the same as in the two-phase method.

\section{Experiments}

\section{A. Experimental Setup}

The experimental setup, as shown in Fig. 1, is the same as the one reported previously except for the data-acquisition procedures. ${ }^{11}$ A coordinate was given for reference. The optical axis was along the $X$ axis, and the ultrasonic axis was along the $Z$ axis. A diode laser of $690 \mathrm{~nm}$ in wavelength (Melles Griot, 56IMS667) was used as the light source. The coherence length of the laser was $\sim 6 \mathrm{~cm}$ when it was modulated at $1 \mathrm{MHz}$. The laser beam incident upon the sample was $\sim 8 \mathrm{~mm}$ in diameter and had a power of $\sim 11 \mathrm{~mW}$. Ultrasonic waves were generated by a focused ultrasonic transducer (UltranLab, VHP1001-R38) with a 38-mm focal length in water and a $1-\mathrm{MHz}$ central response frequency, and they were coupled into the tissue sample through water in which the sample was partially immersed. The focal zone of the ultrasonic waves was $\sim 2 \mathrm{~mm}$ in diameter and $\sim 20 \mathrm{~mm}$ in length. The peak pressure at the focus was $\sim 10^{5} \mathrm{~Pa}$, which is below the damage threshold for biological tissues. The light transmitted through the tissue generated speckle patterns, which were detected by a 12-bit digital CCD camera (Dalsa, CA-D1-0256T) with $256 \times 256$ pixels. The 
exposure time of the CCD camera was selected such that sufficient photons were collected. An iris was placed behind the sample to control the speckle size to approximately equal the CCD pixel size, which was $\sim 16 \mu \mathrm{m}$. Two function generators (Stanford Research Systems, DS345) were used to drive the ultrasonic transducer and to modulate the diode laser, respectively. An $\mathrm{rf}$ amplifier (ENI, 325LA) was applied to amplify the signals driving the transducer. The two function generators shared the same time base for synchronization. The function generators and the CCD camera were controlled with a computer.

\section{B. Experimental Results and Discussion}

In the experiment, the source-synchronized lock-in technique was applied. The diode laser and the ultrasonic transducer were modulated at the same frequency of $1 \mathrm{MHz}$. The phase delay was set to a sequence of $0^{\circ}, 90^{\circ}, 180^{\circ}$, and $270^{\circ}$ or one of $0^{\circ}, 180^{\circ}$, $90^{\circ}$, and $270^{\circ}$. The different sequences did not show differences in the final results.

The host tissue samples were chicken breast tissues and the buried objects were also chicken tissues-gizzards, which had a larger absorption coefficient and almost the same scattering coefficient as the chicken breast tissues. All of the methods just described were applied to imaging the samples. To obtain a $2 \mathrm{D}$ image, we scanned the tissue sample along the $X$ axis and $Y$ axis.

Figure 2 shows a comparison of the 2D images obtained with the various methods. Figures 2(a) and 2(b) are images obtained with the four-phase method and the three-phase method, respectively. The combination of phase delays of $0^{\circ}, 90^{\circ}, 180^{\circ}$ was used in the three-phase method. Other combinations produced similar results. The sample was 15 $\mathrm{mm}$ thick in the $X$ direction with two gizzard objects buried in the middle plane. The original sizes of the objects were approximately $1.5 \mathrm{~mm} \times 2.5 \mathrm{~mm} \times 7$ $\mathrm{mm}$ along the $X, Y$, and $Z$ axes, respectively. The sizes changed after the sample was pressed with two parallel plates to maintain a uniform thickness in the $X$ direction. In both images, the two objects are clearly seen, which indicates that the three-phase method-with less acquisition time-can generate an image with a quality close to that of the four-phase method.

Figures 2(c) and 2(d) show images obtained with two two-phase methods for the same sample. The images are from the acquisitions of the $0^{\circ}$ and $180^{\circ}$ phase delays: the images from the $90^{\circ}$ and $270^{\circ}$ phase delays are similar and are not shown. The images in Figs. 2(c) and 2(d) are almost the same and are similar to those from the four-phase method. The result justifies the approximations in Eqs. (16) and (17) and Eqs. (19)-(22), and it shows that twophase methods can be used as an alternative to the four-phase method to reduce acquisition time significantly.

Figure 2(e) is an image obtained with the crosscorrelation method. The cross correlation of speckle
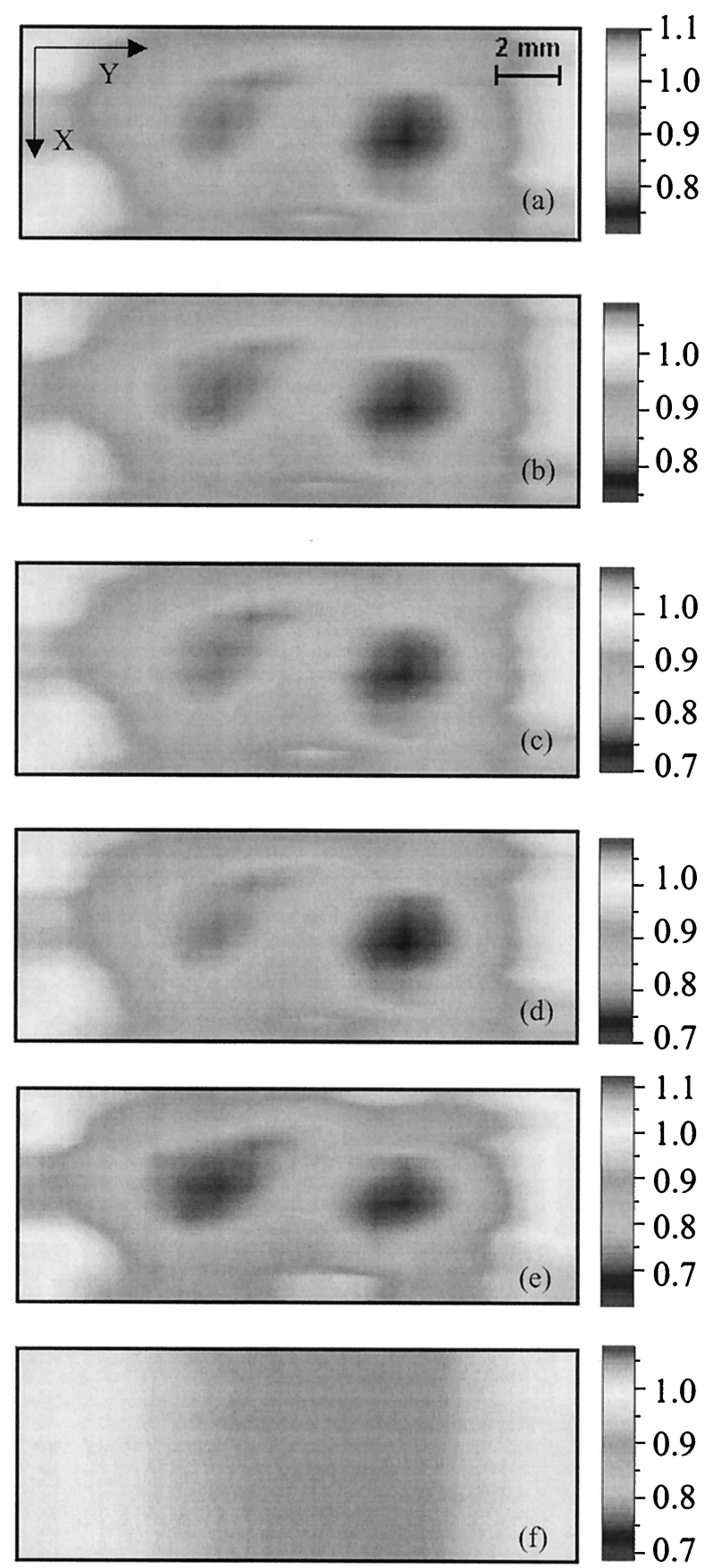

Fig. 2. 2D images of 15 -mm-thick chicken breast tissue in which two gizzard objects were buried. The images were obtained with the (a) four-phase, (b) three-phase, (c) two-phase I, (d) two-phase II, and (e) cross-correlation I methods, and with (f) dc signals.

patterns that were generated with $0^{\circ}$ and $90^{\circ}$ phase delays was studied. The image looks similar to those obtained with other methods.

Figure 2(f) gives an image using the average intensities of speckle patterns as the imaging signals, namely an image from the dc signals $\left(\langle I\rangle=\left\langle I_{\mathrm{dc}}\right\rangle\right)$. The two objects are invisible in this image, whereas they are sharply visible in the images obtained with the modulated data-indicating the efficacy of spatial encoding using the focused ultrasonic beam. 

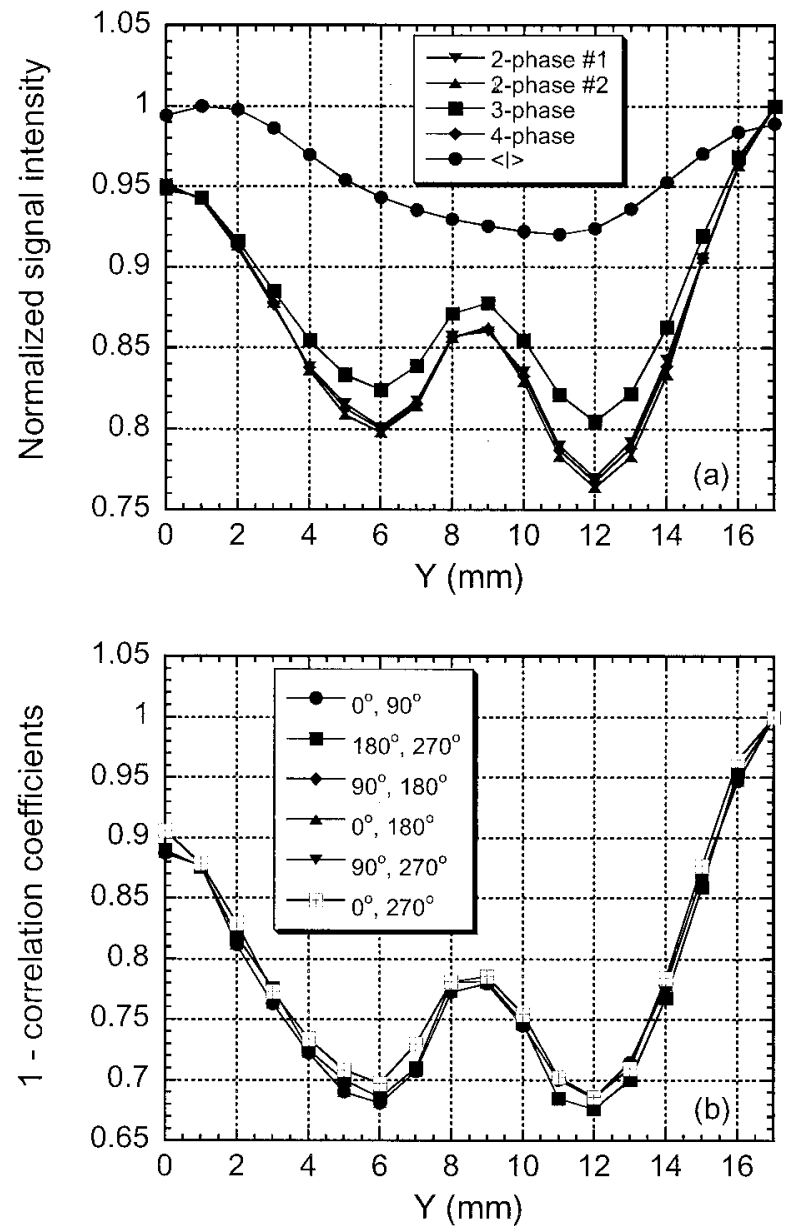

Fig. 3. Comparison of 1D images: (a) obtained from four-phase, three-phase, and two-phase methods, as well as an image from the dc signals; (b) from the cross-correlation coefficients, which were obtained from the various combinations of two different phasedelay acquisitions.

To compare the image qualities in detail, we plotted the 1D images corresponding to the scan line along the $Y$ axis at the center of the sample. Figure 3 (a) shows the 1D images obtained with the four-, three-, and two-phase methods, as well as one from the dc signals. It is apparent that the images obtained with the two-phase methods are almost the same as that obtained with the four-phase method. A detailed comparison shows that the contrast of images obtained with the three-phase method is lower than with the two- or four-phase methods. Figure 3 (b) compares the 1D images obtained with the correlation coefficients calculated from the combinations of any two of the acquisitions of different phase delays. The images from different combinations are similar. Comparing Figs. 3(a) and 3(b) quantitatively, one can see that the contrasts of images obtained with the correlation method are slightly higher.

Because the images from the two-phase methods and the correlation method have qualities similar to those from the four-phase method, which has been used in parallel detection, these methods are supe-

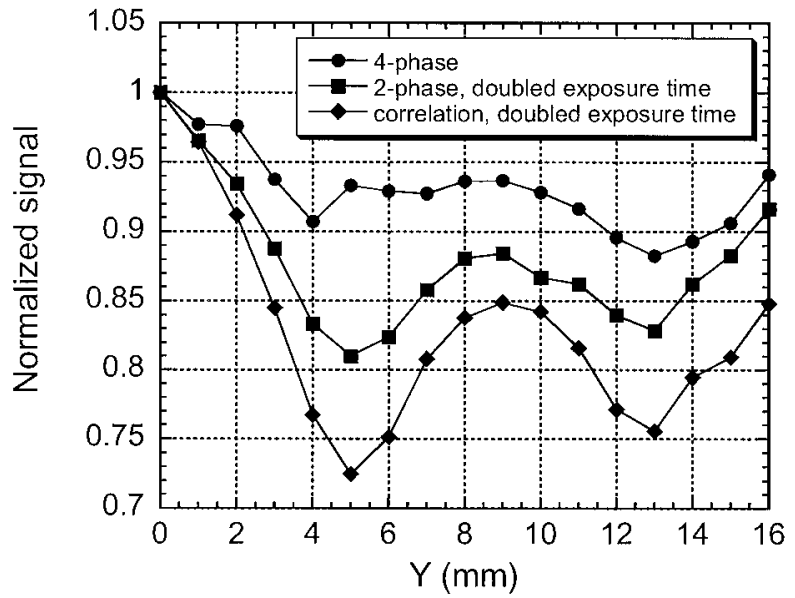

Fig. 4. Comparison of images obtained with the four-phase, twophase, and cross-correlation methods, respectively. The exposure time in the two-phase and cross-correlation methods was doubled.

rior because they have the advantage of requiring half the acquisition time. It is recognized that speckle decorrelation caused by the Brownian motion of scatterers has to be avoided in parallel detection. A correlation time of $400 \mathrm{~ms}$ was measured in our previous experiments with $12-\mathrm{mm}$ thick chicken tissues ${ }^{11}$ and the correlation time was found to decrease with the increase of tissue thickness. The data-acquisition time must be shorter than the correlation time. However, sufficient exposure is needed in the case of low laser power or thick samples. Therefore, the reduction of acquisition time with the two-phase methods or the correlation method is significant.

In terms of the SNR, the two-phase methods and the correlation method yield higher SNRs than the four-phase method. With the same acquisition time, the exposure time of each acquisition in the twophase methods or the correlation method can be two times as long as that in the four-phase method. The increase of exposure time will increase the SNR. Figure 4 shows a comparison of the 1D images obtained with the four-phase method, two-phase method, and cross-correlation method, respectively, where the last two methods had a doubled exposure time. The measurements were made with another chicken tissue sample, which was $\sim 16 \mathrm{~mm}$ thick. It is shown that the SNR improvement with the twophase method or with the cross-correlation method also enhanced the image contrast. The figure shows that the image contrast obtained with the crosscorrelation method was higher than that obtained with the two-phase method, which agrees with the result shown in Figure 3. This implies that the correlation method has higher sensitivity in detecting the ultrasonic modulation.

Moreover, we compared images obtained with our two two-phase methods and with the method of Selb et al. ${ }^{21}$ The comparison shows that the three different two-phase methods produce images with similar qualities. 


\section{Conclusions}

This research extends the study of ultrasoundmodulated optical tomography based on parallel speckle detection. In an effort to improve on the conventional four-phase method, we studied four methods of signal acquisition and analysis. Our imaging experiments utilized all-biological-tissue samples: chicken breast tissues in which two chicken gizzard objects were buried.

A comparison of the resulting images shows that, with the same exposure time for each acquisition, these methods produce images with a quality comparable to those produced by the four-phase method, but with a reduced total acquisition time. Likewise, the exposure time in the two-phase methods or the correlation method can be two times as long as that in the four-phase method. Significant improvements of the SNR, that is, image quality, through the use of the two-phase methods and the cross-correlation method were observed. We conclude that these methods are superior to the four-phase method and should be applied to ultrasound-modulated optical tomography in the future.

This project was sponsored in part by National Institutes of Health Grant R01 CA71980; National Science Foundation Grant BES-9734491; and Texas Higher Education Coordinating Board Grant 0005120123-1999.

\section{References}

1. R. A. Kruger and P. Liu, "Photoacoustic ultrasound: theory," in Laser-Tissue Interaction V, S. L. Jacques, ed., Proc. SPIE 2134A, 114-118 (1994).

2. A. A. Oraevsky, R. O. Esenaliev, S. L. Jacques, and F. K. Tittle, "Laser optic-acoustic tomography for medical diagnostics: principles," in Biomedical Sensing, Imaging, and Tracking Technologies I, R. A. Lieberman, H. Podbielska, and T. VoDinh, eds., Proc. SPIE 2676, 22-31 (1996).

3. C. G. A. Hoelen, F. F. M. de Mul, R. Pongers, and A. Dekker, "Three-dimensional photoacoustic imaging of blood vessels in tissue," Opt. Lett. 23, 648-650 (1998).

4. L.-H. Wang and Q. Shen, "Sonoluminescence tomography of turbid media," Opt. Lett. 23, 561-563 (1998).

5. F. A. Marks, H. W. Tomlinson, and G. W. Brooksby, "Comprehensive approach to breast cancer detection using light: photon localization by ultrasound modulation and tissue characterization by spectral discrimination," in Photon Migration and Imaging in Random Media and Tissues, R. R. Alfano and B. Chance, eds., Proc. SPIE 1888, 500-510 (1993).

6. L.-H. Wang, S. L. Jacques, and X. Zhao, "Continuous-wave ultrasonic modulation of scattered laser light to image objects in turbid media," Opt. Lett. 20, 629-631 (1995).

7. L.-H. Wang and X. Zhao, "Ultrasound-modulated optical tomography of absorbing objects buried in dense tissuesimulating turbid media," Appl. Opt. 36, 7277-7282 (1997).

8. M. Kempe, M. Larionov, D. Zaslavsky, and A. Z. Genack, "Acousto-optic tomography with multiple scattered light," J. Opt. Soc. Am. 14, 1151-1158 (1997).

9. L.-H. Wang and G. Ku, "Frequency-swept ultrasoundmodulated optical tomography of scattering media," Opt. Lett. 23, 975-977 (1998).

10. S. Leveque, A. C. Boccara, M. Lebec, and H. Saint-Jalmes, "Ultrasonic tagging of photon paths in scattering media: parallel speckle modulation processing," Opt. Lett. 24, 181-183 (1999).

11. G. Yao and L.-H. Wang, "Theoretical and experimental studies of ultrasound-modulated optical tomography in biological tissue," Appl. Opt. 39, 659-664 (2000).

12. G. Yao, S. Jiao, and L.-H. Wang, "Frequency-swept ultrasound-modulated optical tomography in biological tissue by use of parallel detection," Opt. Lett. 25, 734-736 (2000).

13. S. Leveque-Fort, "Three-dimensional acousto-optic imaging in biological tissues with parallel signal processing," Appl. Opt. 40, 1029-1036 (2000).

14. A. Lev, Z. Kotler, and B. G. Sfez, "Ultrasound tagged light imaging in turbid media in a reflectance geometry," Opt. Lett. 25, 378-380 (2000).

15. E. Granot, A. Lev, Z. Kotler, and B. G. Sfez, "Detection of inhomogeneities with ultrasound tagging of light," J. Opt. Soc. Am. A 18, 1962-1967 (2001).

16. S. Leveque-Fort, J. Selb, L. Pottier, and A. C. Boccara, "In situ local tissue characterization and imaging by backscattering acousto-optic imaging," Opt. Commun. 196, 127-131 (2001).

17. W. Leutz and G. Maret, "Ultrasonic modulation of multiply scattered light," Physica B 204, 14-19 (1995).

18. G. D. Mahan, W. E. Engler, J. J. Tiemann, and E. Uzgiris, "Ultrasonic tagging of light: theory," Proc. Natl. Acad. Sci. USA 95, 14,015-14,019 (1998).

19. L.-H. V. Wang, "Mechanisms of ultrasonic modulation of multiply scattered coherent light: an analytic model," Phys. Rev. Lett. 87, 1-4 (2001).

20. L.-H. V. Wang, "Mechanisms of ultrasonic modulation of multiply scattered coherent light: a Monte Carlo model," Opt. Lett. 26, 1191-1193 (2001).

21. J. Selb, S. Leveque-Fort, L. Pottier, and C. Boccara, "Setup for simultaneous imaging of optical and acoustic contrasts in biological tissues," in Biomedical Optoacoustics II, Alexander A. Oraevsky, ed., Proc. SPIE 4256, 200-207 (2001).

22. J. W. Goodman, "Statistical properties of laser speckle patterns," in Laser Speckle and Related Phenomena, Vol. 9 of Topics in Applied Physics, J. C. Dainty, ed. (Springer-Verlag, Berlin, 1984). 